\title{
Drosophila Chitinase 2 is expressed in chitin producing organs for cuticle formation
}

\author{
Yanina-Yasmin Pesch a, b, Dietmar Riedel c, Matthias Behr ${ }^{\mathrm{a}, \mathrm{b}, *}$ \\ a Institute for Biology and Sächsischer Inkubator für klinische Translation (TRM/SIKT), University of Leipzig, 04103 Leipzig, Germany \\ ${ }^{\mathrm{b}}$ Life \& Medical Sciences Institute (LIMES), University of Bonn, 53115 Bonn, Germany \\ ${ }^{\mathrm{c}}$ Max Planck Institute for Biophysical Chemistry, Electron Microscopy Group, 37077 Göttingen, Germany
}

\section{A R T I C L E I N F O}

\section{Article history:}

Received 19 May 2016

Received in revised form

1 November 2016

Accepted 2 November 2016

Available online 19 November 2016

\section{Keywords:}

Arthropod

Epidermis

Epithelia

Gut

Salivary gland

Tracheal system

\begin{abstract}
A B S T R A C T
The architecture of the outer body wall cuticle is fundamental to protect arthropods against invading pathogens and numerous other harmful stresses. Such robust cuticles are formed by parallel running chitin microfibrils. Molting and also local wounding leads to dynamic assembly and disassembly of the chitin-matrix throughout development. However, the underlying molecular mechanisms that organize proper chitin-matrix formation are poorly known. Recently we identified a key region for cuticle thickening at the apical cell surface, the cuticle assembly zone, where Obstructor-A (Obst-A) coordinates the formation of the chitin-matrix. Obst-A binds chitin and the deacetylase Serpentine (Serp) in a core complex, which is required for chitin-matrix maturation and preservation. Here we present evidence that Chitinase 2 (Cht2) could be essential for this molecular machinery. We show that Cht2 is expressed in the chitin-matrix of epidermis, trachea, and the digestive system. There, Cht2 is enriched at the apical cell surface and the dense chitin-matrix. We further show that in Cht2 knockdown larvae the assembly zone is rudimentary, preventing normal cuticle formation and pore canal organization. As sequence similarities of Cht 2 and the core complex proteins indicate evolutionarily conserved molecular mechanisms, our findings suggest that Cht2 is involved in chitin formation also in other insects.
\end{abstract}

() 2016 Elsevier Ltd. All rights reserved.

\section{Introduction}

The epidermal and tracheal cuticles are the outermost physical barriers that protect insects against a harmful environment and are required to maintain homeostasis of body fluids. However, numerous events, such as metamorphosis, tissue remodeling and wounding lead to dynamic changes in the cuticle structure (Galko and Krasnow, 2004; Moussian and Uv, 2005; Matsuda et al., 2007; Jaspers et al., 2014; Pesch et al., 2016). Any impairment of cuticle assembly and degradation processes is fatal to the protection system resulting in insects' death (Petkau et al., 2012; Pesch et al., 2015).

The insect cuticle comprises three main layers. The outermost epicuticle and the envelope may both play roles to provide a water barrier (Gibbs, 1998; Shaik et al., 2011; Curtis et al., 2013), protecting

Abbreviations: asp, anterior spiracles; Cht, Chitinase; ep, epidermis; fg, foregut; hi, hindgut; Idgf, Imaginal disc growth factor; Knk, Knickkopf; mg, midgut; Obst-A, Obstructor-A; psp, posterior spiracles; pv, proventriculus; Serp, Serpentine; sg, salivary glands; ts, tracheal system; vm, ventral midline.

* Corresponding author. Institute for Biology and Sächsischer Inkubator für klinische Translation (TRM/SIKT), University of Leipzig, 04103 Leipzig, Germany.

E-mail address: matthias.behr@uni-leipzig.de (M. Behr). insects against swelling and dehydration (Jaspers et al., 2014). The largest and most prominent part is formed by the lamellate procuticle (protein-chitin-matrix) that dramatically thickens during late larval development. In Drosophila final larval stage the procuticle increases from approximately six to more than fifty lamellae, with almost one lamella forming per hour (Kaznowski et al., 1985; Pesch et al., 2015). Underlying molecular mechanisms that regulate chitin-matrix organization at the assembly zone and thickening of the procuticle, finally control cuticle properties, such as stability and resistance against mechanical stresses and invading pathogens (Petkau et al., 2012; Pesch et al., 2015).

New cuticle material is deposited at the apical cell surface (Merzendorfer, 2006; Moussian et al., 2015), where the chitinmatrix becomes organized into a compact and lamellar structure (Moussian, 2013). Newly synthesized chitin-polymers, consisting of $\beta$-1,4-linked $\mathrm{N}$-acetylglucosamines variable in length and diameter, undergo self-assembly into chitin-nanofibers that may serve as founding structure for chitin-matrices (Merzendorfer, 2006; Chandran et al., 2016). Local cuticular transpiration may affect the fine-tuning of cuticle properties (Klocke and Schmitz, 2011). However, resembling morphologies of chitin-matrices and the 
correlating mechanical and physical properties (Vincent and Wegst, 2004; Chandran et al., 2016) indicate the requirement of conserved genetic mechanisms among arthropod species that control extracellular matrix formation.

Nearly thirty years ago it has been discussed that the assembly zone (synonyms are deposition zone/adhesion zone) is a morphological stable and permeable matrix at the cell surface, through which all chitin precursors pass (Wolfgang et al., 1987). Recently, it was shown in Drosophila larvae that assembly and formation of cuticular layers occurs in the assembly zone at the apical cell surface (Pesch et al., 2015). There chitin is bound by a core complex of proteins that coordinate chitin-matrix assembly, packaging and finally cuticle stability (Petkau et al., 2012). The obstructor-A (obstA) gene, a member of the obstructor multigene family, encodes a small protein with three Chitin-Binding-Domains type 2 (CBD2) (Behr and Hoch, 2005; Petkau et al., 2012) and belongs to the conserved cuticular proteins analogous to peritrophins (CPAP) (Jasrapuria et al., 2010, 2012; Willis, 2010; Dittmer et al., 2015; Tetreau et al., 2015). The obst- $A$ gene is expressed in cells of cuticle forming organs (Behr and Hoch, 2005) and its gene product is secreted towards the extracellular matrix. There it binds chitin to form a scaffold that recruits and localizes the deacetylase Serpentine (Serp) (Luschnig et al., 2006; Wang et al., 2006) in the matrix, which matures and improves properties of the forming matrix in the tracheal lumina (Petkau et al., 2012) as well as in the epidermis (Pesch et al., 2015). In addition, the GPI-linked Knickkopf (Knk) protein is required at the Obst-A scaffold (Petkau et al., 2012; Pesch et al., 2015), where Knk organizes cuticle differentiation (Moussian et al., 2006) and probably protects the newly synthesized cuticles against chitinolytic attack (Chaudhari et al., 2011). Finally, loss of Obst-A function causes severe cuticle defects in larvae, such as absence of the assembly zone and a deformed procuticle structure leading to an impaired cuticle barrier, which is fragile against environmental stresses (Petkau et al., 2012; Pesch et al., 2015).

Altogether, the new cuticular material contains chitin nanofibrils in an amorphous matrix of proteins (Zhu et al., 2016), which require the Obst-A mediated core complex for chitin-matrix formation. Here we discuss a novel and unexpected molecular mechanism of chitin-matrix formation that involves the Chitinase 2 (Cht2) protein. Chitinases belong to the glycosylhydrolases which are widely spread in all kingdoms, including bacteria, plants and animals (Adrangi and Faramarzi, 2013). In insects the class II glycosylhydrolase (Glyco 18) domain is characteristic for the large gene family consisting of Chitinases (Cht) and Chitinase-like imaginal disc growth factors (idgf). The insect Chts may act like endochitinases which digest the $\beta-1,4$-linkages of polymeric chitin. Their main function is probably the turnover of chitin-containing extracellular matrices in the cuticle and peritrophic matrix during molting of insects (Arakane and Muthukrishnan, 2010). The Glyco 18 domain sequence is well-conserved among insects and its chitinolytic activity has been chemically characterized for a number of Chitinases in Drosophila and Tribolium (Zhu et al., 2008a, 2008b; Arakane and Muthukrishnan, 2010). Thus, it is believed that Chts may be key enzymes for chitin degradation but, since there are many members in insects with diverse roles during development, their precise function is not understood.

Ten Cht and six idgf genes have been identified in the Drosophila genome (Zhu et al., 2008a; Zhang et al., 2011a; Pesch et al., 2016). A first systematic RNAi-based knockdown screen of individual Drosophila Cht and idgf genes showed high mortality rates and cuticle molting defects in larvae and pupae upon Cht2 RNA reduction. In addition, during larval intermolt stages the Cht2 knockdown led to a destabilized and fragile epidermal cuticle that failed to resist mechanical stresses and did not protect larvae from invading pathogens (Pesch et al., 2016). In this study we systematically investigated the Cht2 protein expression and localization pattern. Cht2 is expressed in ectodermal cuticle forming organs, such as epidermis, trachea and the digestive system, already from late embryonic stages onwards. At the subcellular level we identified the Cht 2 protein to be enriched in the assembly zone of the body wall cuticle and scattering along the lamellate chitin-matrix of the procuticle. Reduction of the Cht2 protein upon RNAi-knockdown results in the absence of the characteristic assembly zone structure preventing the thickening of the lamellate procuticle and the formation of normal pore-canal like structures in Drosophila larvae.

\section{Material and methods}

\subsection{Fly work}

Vienna (Austria)/Bloomington (USA) stock centers (http:// stockcenter.vdrc.at/control/main and http://flystocks.bio.indiana. edu/): $w 1118$ (referred to as wild type (wt)), 69B-Gal4 line, btl-Gal4 line, UAS-RNAi-Cht2 line (Vienna stock center), UAS-Cht2 line (Tonning et al., 2005). For knockdown analysis, 69B-Gal4 flies were mated with UAS-RNAi-Cht2 flies and for overexpression, btl-Gal4 flies were mated with UAS-Cht2 flies as described in (Pesch et al., 2016).

\subsection{Antibodies and immunofluorescent assays}

Embryo preparation and fixation with $4 \%$ formaldehyde and immunofluorescent labelling was performed as described in (Stümpges and Behr, 2011). Wandering third instar larvae were dissected and organs were fixed $1 \mathrm{~h}$ in $4 \%$ formaldehyde in PBS (Wingen et al., 2009). For sections wandering third instar larvae were fixed overnight at $4{ }^{\circ} \mathrm{C}$ in $4 \%$ paraformaldehyde and embedded in JB-4 resin (Polysciences, USA, Warrington, USA). Sections of $7 \mu \mathrm{m}$ were cut using an Ultracut E (Reichert-Jung, Solms, Germany). Immunofluorescent staining was performed as described (Pesch et al., 2015).

The anti-Cht 2 antibody against the peptide WDPQTSQVLAKSERN (amino acid 335 to 349 of Cht2) was generated in a guinea pig and purified from the serum by Pineda (Berlin, Germany). The Cht2 antibody was used 1:50 (embryo whole mounts and larval sections) and 1:100 (larval organs after dissection) for immunofluorescent labelling and 1:5000 for western blot analysis. For peptide competition the Cht 2 antibody was treated $1 \mathrm{~h}$ with the anti-Cht 2 peptide $(0.5 \mu \mathrm{g} / \mu \mathrm{l}$ antibody) as described in Wingen et al. (2009). For labelling studies embryos were incubated $72 \mathrm{~h}$ at $4{ }^{\circ} \mathrm{C}$ with the $\mathrm{Cht} 2$ antibody and larval sections and organs overnight at $4{ }^{\circ} \mathrm{C}$. The following primary antibodies were used: $\alpha$-Spectrin (3A9, 1:10, mouse, DSHB, Iowa, USA), Obst-A (1:300; rabbit (Petkau et al., 2012)). For detection of the primary antibody, secondary antibodies coupled to fluorophores (Dianova, Hamburg, Germany and JacksonImmuno, Baltimore, USA) were used together with the Alexa633-conjugated wheat germ agglutinin (WGA) (1:250; Molecular probes, Invitrogen, Carlsbad, USA). WGA selectively recognizes $N$-acetylneuraminacid and $\mathrm{N}$-acetylglucosamine sugar residues at the chitin-matrix and plasma membrane surfaces. Embryos and larval sections were mounted in Vectashield (H-1500, DAPI, Vector Laboratories, Burlingame, USA). Dissected organs were mounted in Fluoromount with DAPI (SouthernBiotech, Birmingham, USA).

Confocal assays were performed with a LSM710 and a LSM780Airyscan (Zeiss, Jena, Germany; $25 \times$ and $63 \times$ LCI Plan Neofluar objectives). We used standard settings, the pinhole "airy unit 1" (ZEN 2010 and ZEN 2011), optimal filter settings and sequential scan mode to avoid cross-talk. Images were cropped with Adobe Photoshop CS6 and figures were designed with Adobe Illustrator CS6. 


\subsection{Ultrastructure analysis}

Drosophila second and third instar larvae were placed on a $150 \mu \mathrm{m}$ flat embedding specimen holder (Engineering Office M. Wohlwend $\mathrm{GmbH}$, Sennwald, Switzerland) and frozen using 1hexadecene in a Leica HBM 100 high pressure freezer (Leica Microsystems, Wetzlar, Germany) according to the described method (Pesch et al., 2015). Images were taken by a Philips CM120 electron microscope (Philips Inc., Eindhoven, The Netherlands) using a F416 CMOS slow scan CCD camera (TVIPS, Gauting, Germany).

\subsection{Western blot analysis}

Embryos, larvae, pupae and adult freshly eclosed flies, respectively, were lysed in RIPA Buffer supplemented with $1 \times$ Protease Inhibitor Cocktail (Roche, Basel, Switzerland) using the Precellys Homogenizer (Cayman Chemical, Ann Arbor, USA). Protein concentration was determined with the Pierce BCA Protein Assay Kit (Thermo Scientific, Waltham, USA) and samples ( $8 \mu \mathrm{l})$ with equalized protein concentration $(1.9 \mathrm{mg} / \mathrm{ml})$ were subjected to SDS-PAGE and transferred to an Immobilon-P PVDF membrane (Merck Millipore, Darmstadt, Germany). Successful transfer of proteins to the membrane was tested by PonceauS staining. After blotting, the membrane was blocked for $2 \mathrm{~h}$ with $5 \%$ milk in TBST (TBS with $0.2 \%$ Tween-20) and incubated overnight $\left(4^{\circ} \mathrm{C}\right)$ with anti-Cht2 (1:5000). A secondary antibody conjugated with Horseradish peroxidase (HRP) (anti-guinea pig, Santa Cruz, Dallas, USA) was used for chemi-luminescent detection with Pierce ECL Western Blotting Substrate (Thermo Scientific, Waltham, USA).

\section{Results}

So far the knowledge about Cht 2 expression and localization in Drosophila and its orthologs in other insects is very limited. In order to investigate the Cht2 protein (484 amino acids predicted), we generated a polyclonal antibody recognizing a peptide stretch of fifteen amino acids [WDPQTSQVLAKSERN] at position 335-349 which is part of the Glyco 18 domain but is exposed to the outside of the protein (Fig. 1A and B). The antibody was purified out of the serum and first tested by western blotting, where it detects a single prominent band matching well to the predicted molecular mass of $54 \mathrm{kDa}$. This prominent band was present in protein extracts from embryos, larvae, pupae and adults (Fig. 1C) which is in good correlation with the fact that the Cht2 gene is expressed throughout development (Pesch et al., 2016).

\subsection{Cht2 expression and extracellular localization in embryos and larvae}

Next, we investigated the Cht2 protein expression by immunofluorescent co-labelling studies during late embryogenesis. As co-markers $\alpha$-Spectrin was used for visualizing cell membranes and wheat germ agglutinin (WGA) for marking cell surfaces and the chitin-matrix. First Cht2 staining was observed in the cuticle forming organs starting with stage 13 in the fore- and hindgut, later on in the tracheal system and finally in the epidermis and the spiracles. Additionally, we detected enriched expression in the embryonic salivary glands (Fig. 2A and B). Negative as well as positive control experiments, such as peptide competition, ectopic Cht2 gene expression, and RNAi based knockdown of the Cht2 gene by the use of the UAS-GAL4 system, demonstrated that the antibody specifically recognizes the Drosophila Cht2 protein (Fig. 2C). In summary, our findings suggest the requirement of Cht 2 in the cuticle forming organs of late embryos.
In order to understand the larval Cht 2 expression pattern we dissected third instar larvae and prepared the organs for immunofluorescent antibody-labelling (Fig. 3). $\alpha$-Spectrin was used as co-marker for detecting cell membranes, WGA for visualizing cell surfaces and Obst-A for chitin-matrix identification. This analysis showed Cht2 staining in cells of the tracheal system (Fig. 3A), the foregut and midgut (Fig. 3B) and the posterior spiracles (Fig. 3C), which all produce chitin-matrices. In addition, we observed Cht2 staining in the small salivary glands cells (Fig. 3D) and in the cells of imaginal discs (Fig. 3E). It is of note that Cht2 signals in third instar larvae were diminished in negative controls upon peptide competition and RNAi based knockdown of the Cht2 gene. Our findings show that Cht 2 is present in the cuticle forming organs of third instar larvae leading to the question where Cht2 is localized in the cuticle. This was further addressed by immunofluorescent colabelling of Cht 2 with WGA in the epidermal body wall cuticle of wandering third instar larvae. Thin sections of such third instar
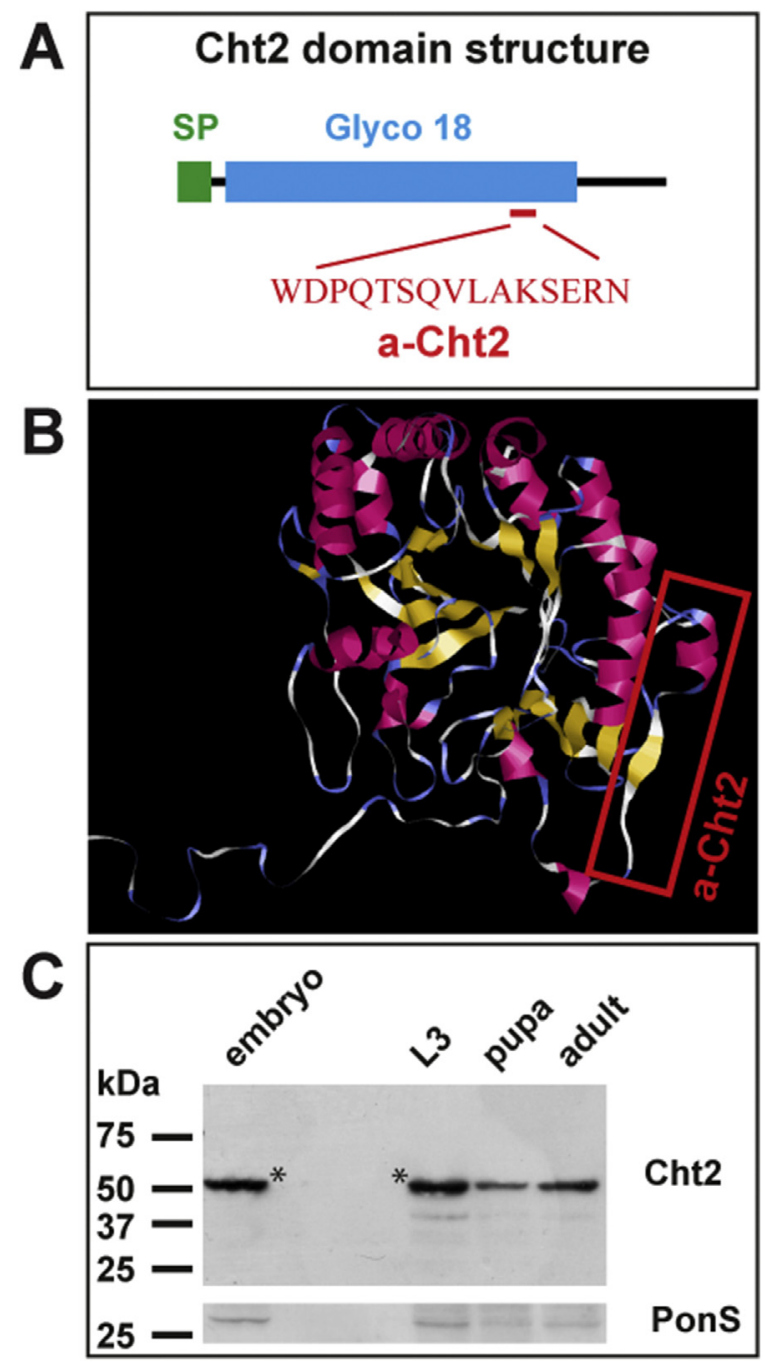

Fig. 1. Cht2 is a Glyco18 domain protein expressed throughout Drosophila lifetime. (A) The cartoon illustrates Cht2 domains predicted by the SMART tool (EMBL, Heidelberg). The signal peptide is indicated in green, the Glyco 18 domain (amino acid 41-389) is shown in blue. The red line marks the epitope region recognized by the Cht 2 antibody (sequence is indicated, amino acids 335-349). (B) The predicted Cht2 topology is visualized with RasMol (2.7.5.2; openrasmol.org) indicating the exposed epitope peptide recognized by the Cht 2 antibody. (C) Immunoblots using the Cht 2 antibody show a predominant signal (asterisks) correlating well with the predicted Cht 2 mass $(54 \mathrm{kDa})$ of total protein extracts of embryos, third instar larvae, pupae and adults. The PonceauS (PonS) control confirms presence of protein extracts in the different lanes. 
larvae can be used for subcellular analysis of the cuticle organization. There WGA was strongly enriched at the apical cell surface and detected the compact chitin lamellae of the procuticle (Pesch et al., 2015). Our stainings showed co-enrichment of Cht2 staining with WGA at the apical cell surface, where the assembly zone is located. In addition, Cht2 was observed at the compact chitin-lamellae (Fig. 3F). Our findings show that Cht 2 is present in the newly synthesized chitin-matrix of the assembly zone, as well as in the compact chitin-lamellae of the procuticle. It further shows that Cht2 is found in the cuticle of larvae during intermolt stages.

\subsection{Defective cuticle formation in Cht2 knockdown larvae}

The epidermal cuticle of third instar larvae is a model to study molecular mechanisms of cuticle formation during larval intermolt stage (Pesch et al., 2015, 2016). Then body wall cuticle undergoes massive thickening by increasing the number of chitin-lamellae of the procuticle (Kaznowski et al., 1985). The underlying molecular mechanism involves the Obst-A scaffold which accumulates at the apical cell surface for organizing chitin-matrix assembly and maturation in the cuticle assembly zone together with Serp and probably with Knk (Pesch et al., 2015). The assembly zone is

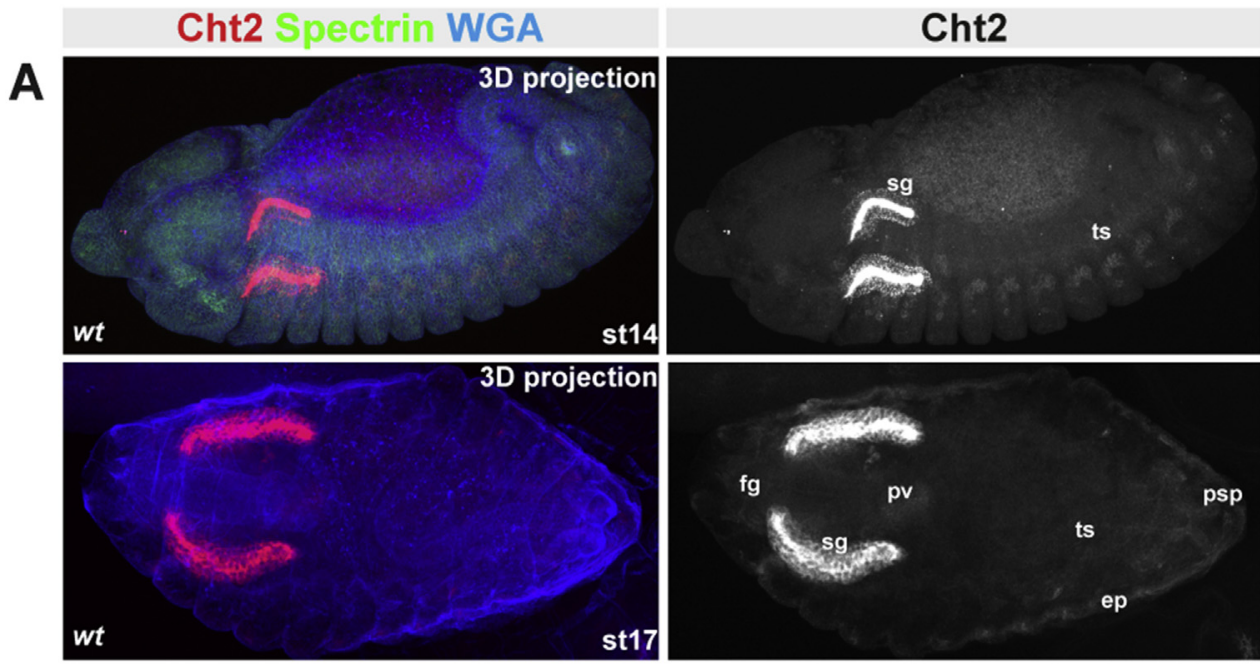

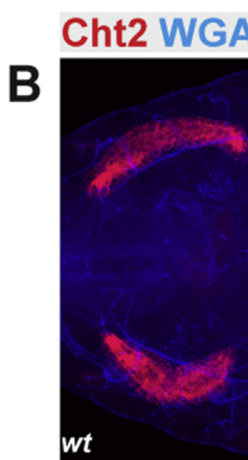

Cht2

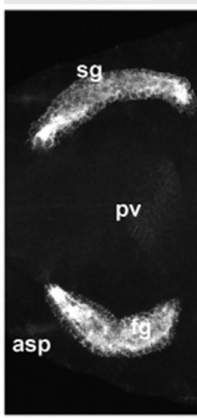

Cht2 Spectrin WGA

C

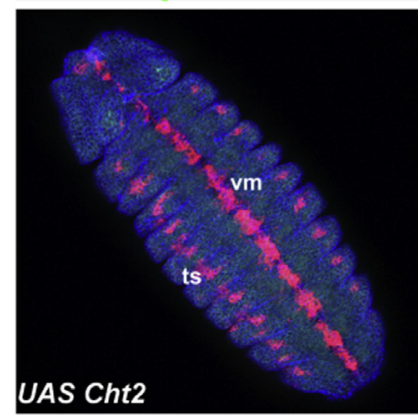

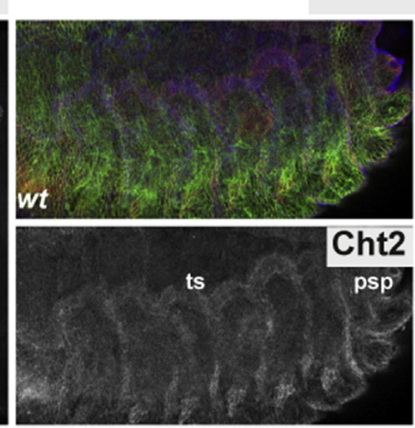

Cht2 Spectrin WGA
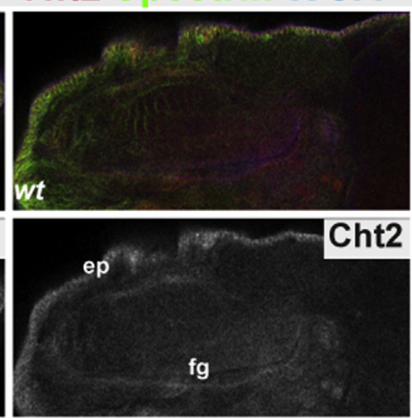

Cht2 Spectrin WGA

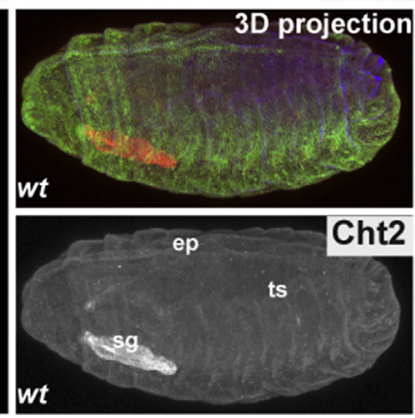

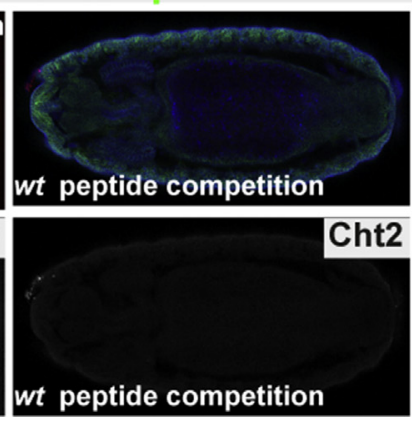

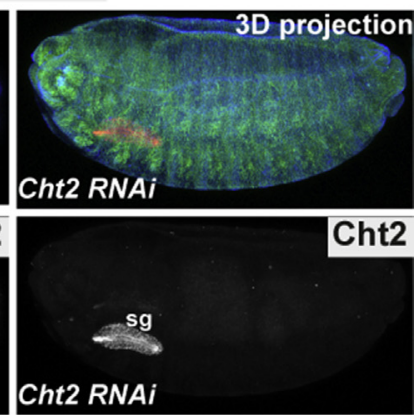

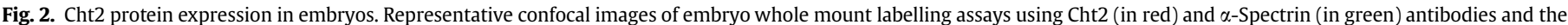

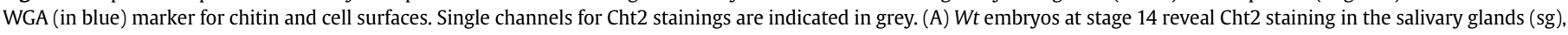

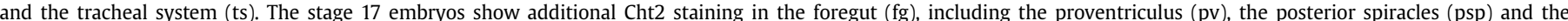



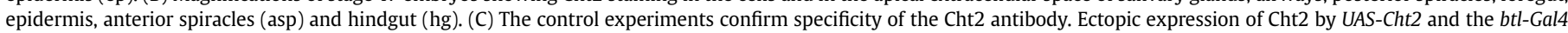

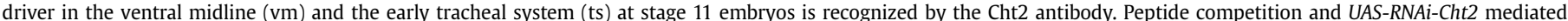

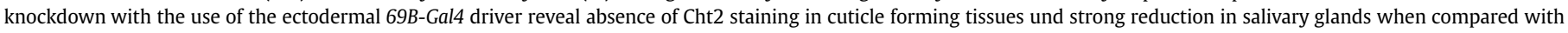
$w t$ embryos. Note: for comparison experiments were simultaneously performed with stage 16 embryos and taken with identical confocal settings ( $\mathrm{n}>20$ embryos). 
Cht2 Obst-A WGA
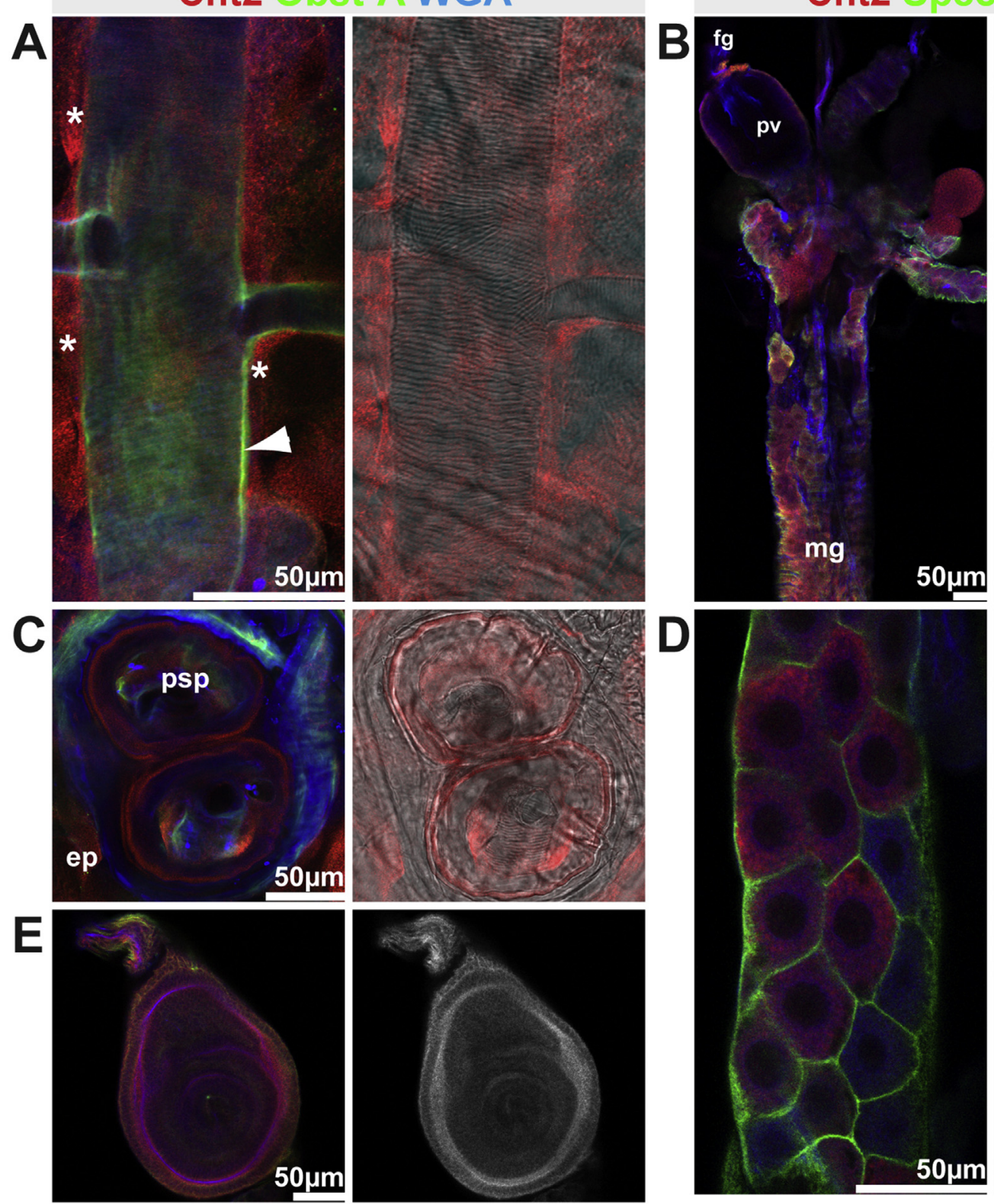

Cht2 Spectrin WGA

\section{D}

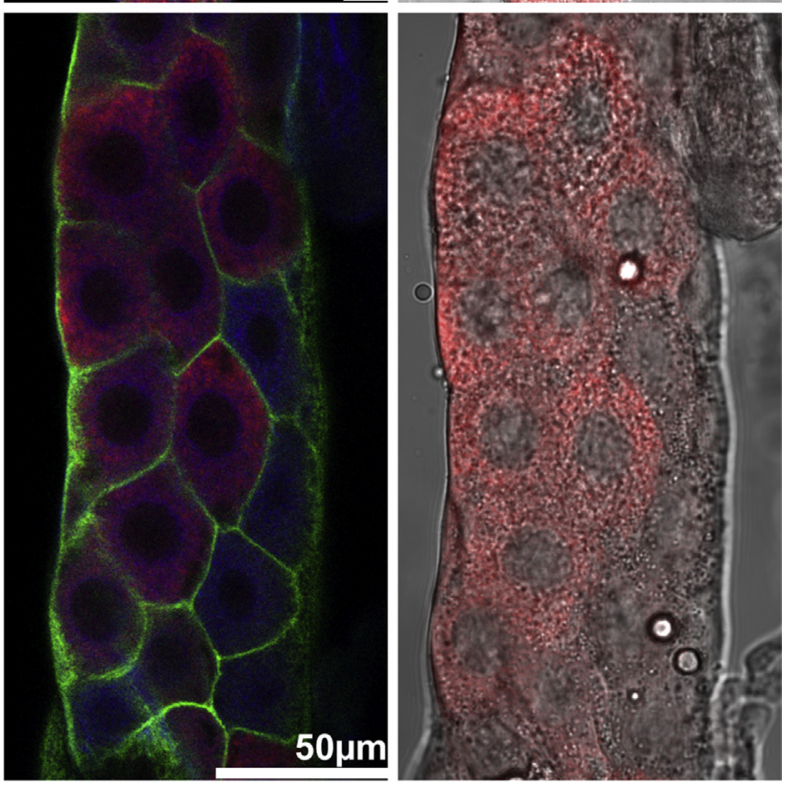

\section{Cht2 WGA}
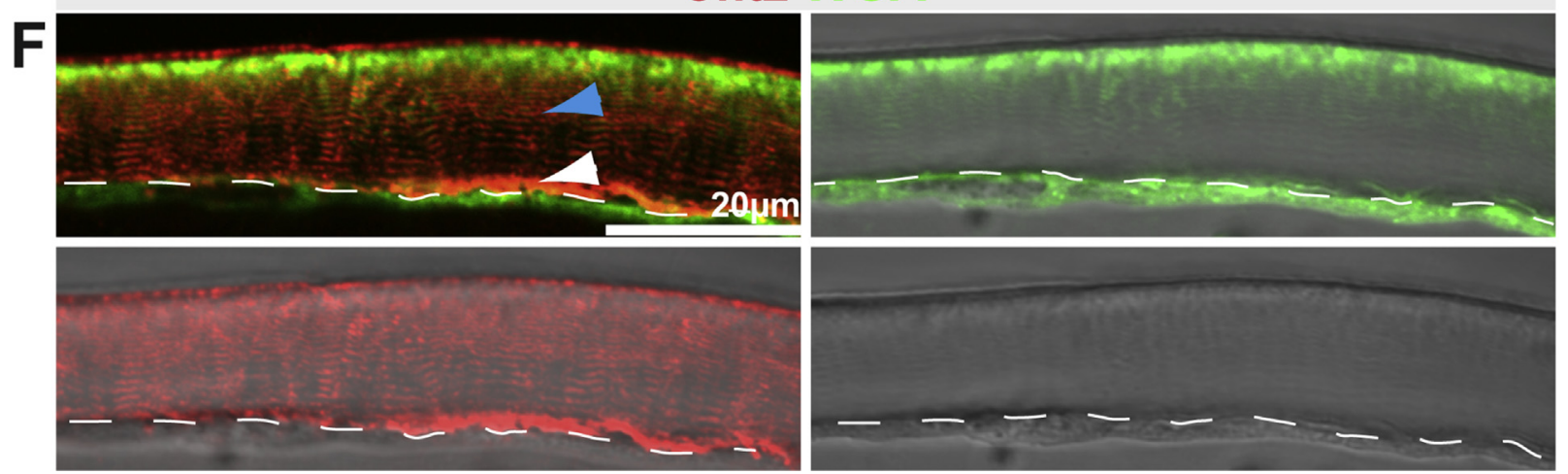

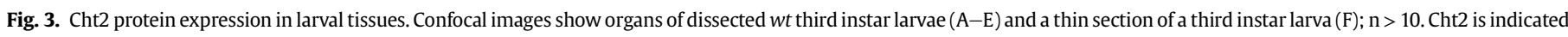

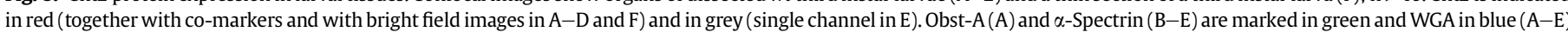

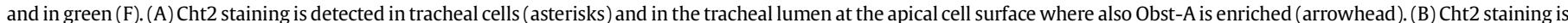

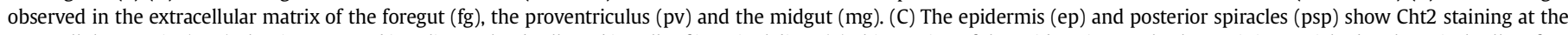

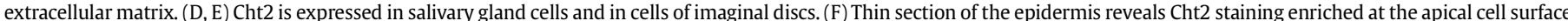

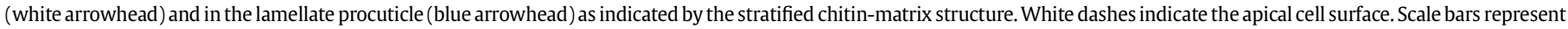
$50 \mu \mathrm{m}(\mathrm{A}-\mathrm{E})$ and $20 \mu \mathrm{m}(\mathrm{F})$. 
localized between the apical cell surface and chitin-matrix lamellae of the procuticle. Obst-A and Serp, which are normally enriched in the cuticle assembly zone, appeared to be mislocalized upon RNAi based Cht2 knockdown (Pesch et al., 2016). Cht2 gene function can be efficiently knocked down by using the UAS-GAL4 system for epidermal Cht2 RNAi expression (Pesch et al., 2016). Ultrastructure images of late wild type third instar larvae enabled the visualization of a zone with less condensed and less ordered chitin-fibrils, the assembly zone, which is localized between the apical cell surface and chitin-matrix lamellae of the procuticle (Fig. 4). In contrast, late third instar Cht2 knockdown larvae showed defective cuticle organization. The assembly zone was diminished and thereby defective chitin-lamellae-like structures of the procuticle reached to the apical cell surface (Fig. 4B).

Pore-canals have been described as structures passing through the lamellate procuticle. Connected to the epithelial cells they may transport wax or cuticle material to the outermost layers (Locke, 1961). It has been discussed that pore-canal formation depends on the chitin-matrix organization (Locke, 1961; Noh et al., 2014), suggesting that any disarrangements in the chitin-lamellae may interfere with the organization of canals. We addressed this by analyzing second and third instar larvae of wild type ( $w$ ) and Cht2 knockdown mutants. Second instar $w t$ larvae show pore canal-like structures running through the lamellate procuticle towards the epicuticle and envelope (Fig. 5A, left panel). The pore canals of Cht2 knockdown mutant larvae appeared in wt-like distribution (Fig. 5A, right panel). This is different in the third instar larvae, when cuticle thickening occurs due to massive chitin-matrix production and formation of numerous chitin-lamellae. In $w t$ pore canals appear as dense grey shadow like structures (Fig. 5C, left inset) that were found running evenly across the epicuticle reaching into the outermost two lamella of the procuticle (Fig. 5B and C, left panel). At the same age Cht2 knockdown mutant larvae showed mis-distributed pore canal structure, which appeared to be fragmented and mis-distributed throughout the entire chitin-matrix (Fig. 5B and C, right panel). The most likely width of the pore canals appeared electron translucent (Fig. 5C, right inset). These findings indicate that appearance and architecture of the pore canals is defective in third instar Cht2 knockdown larvae when wt animals normally produce massive amounts of cuticle-material for chitin-matrix thickening. The Cht2 mutant pore canal-like structures appeared translucent and not dense indicating that canal is not filled with material, suggesting
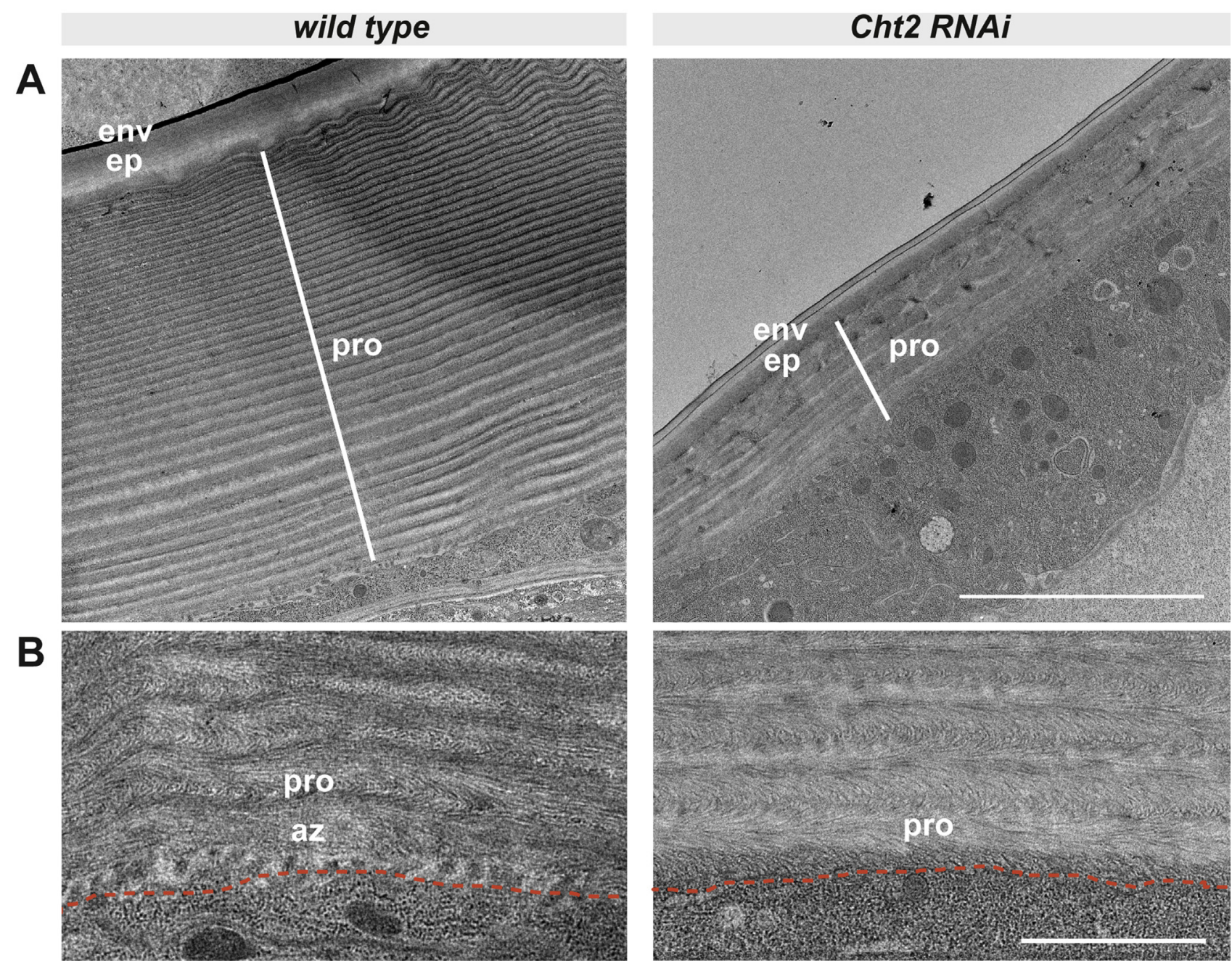

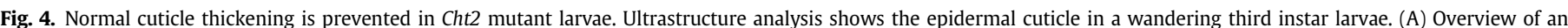

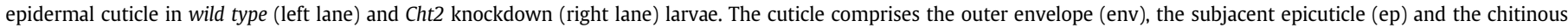

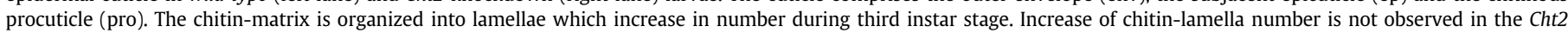

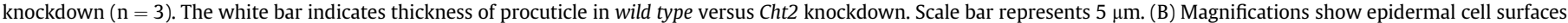


et al., 2016). Scale bar indicates $1 \mu \mathrm{m}$. 


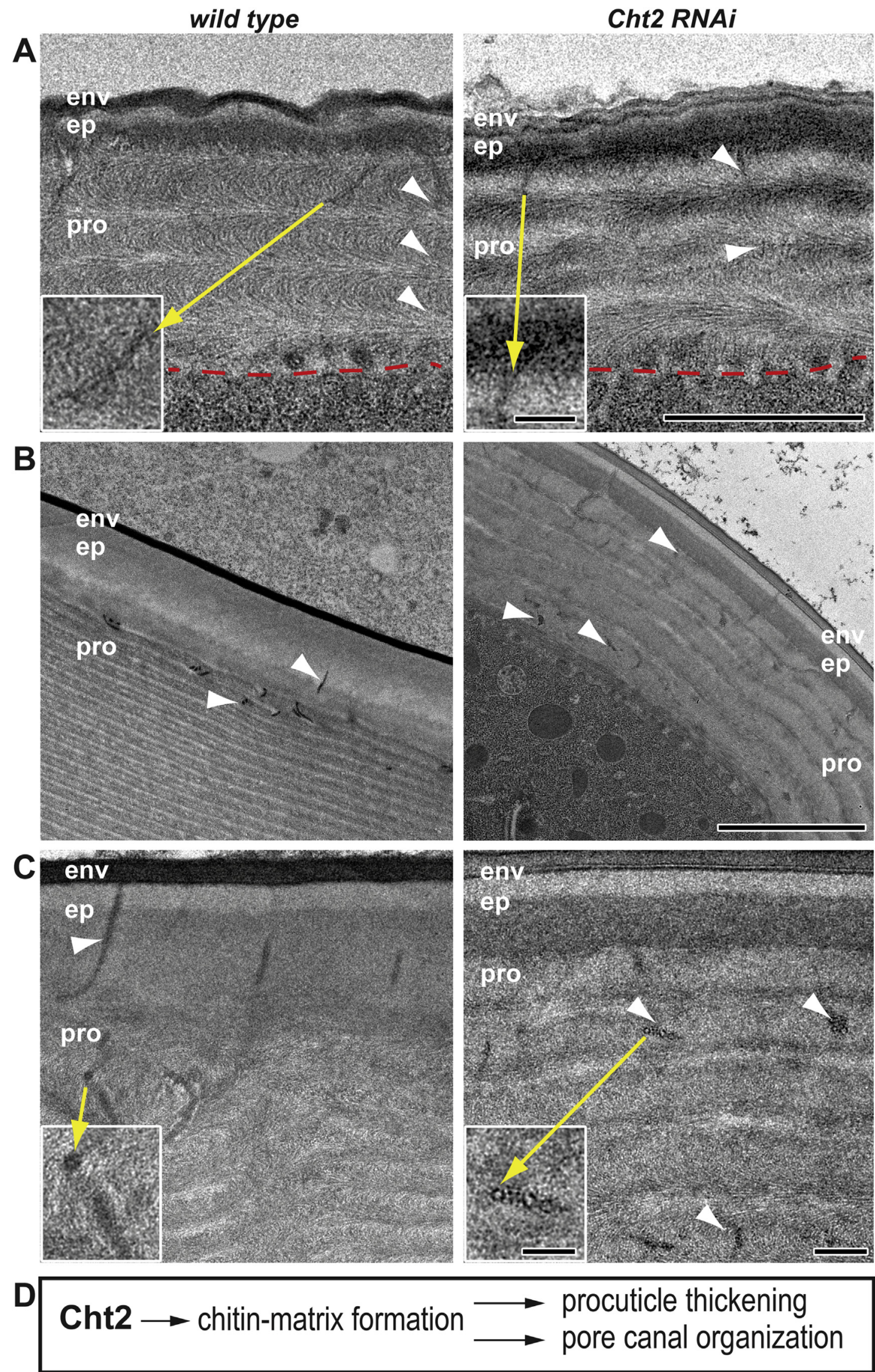


that the normal transport function must be impaired. These findings indicate that the lamellate chitin-matrix architecture is defective in the Cht 2 mutant larvae and thus may have an impact on the function of the outer epicuticle and envelope wax layer.

\section{Discussion}

We investigated the expression and localization pattern of the Drosophila Cht2 protein in embryos and larvae. This pattern is consistent with expression of Cht genes found in many other insects (Zhu et al., 2016). Studies from Tribolium castaneum revealed expression of Cht5, Cht7 and Cht10 genes in the epidermis and possibly in the tracheal system, whereas many other Cht genes are expressed in the midgut (Zhu et al., 2008c). This indicates diverse roles among the family members of Tribolium Cht genes. Similarly, the Anopheles gambiae Chts have diverse expression patterns and many, including the $\mathrm{Ag}$-Cht2, showed various levels of expression throughout development (Zhang et al., 2011b). It has been shown that Drosophila idg genes are active in yolk cells and in the fatbody during mid embryogenesis. In third instar larvae idgfs are expressed in fatbody, ring gland, lymph glands, central brain and in variable patterns in imaginal discs, salivary glands and gut (Kawamura et al., 1999). In contrast, expression data for Drosophila Cht genes are poorly known.

Recently, we investigated relative RNA expression levels of Drosophila Cht and idgf genes throughout development. This showed that most idgf genes are expressed at constant levels, while most Cht genes show time dependent up-regulation during larval and/or pupal stages (Pesch et al., 2016). We also investigated the spatial expression pattern of the gene family members at the end of embryogenesis, when chitin-matrix forms for the first time in a number of organs. This study revealed gene expression in cells of the epidermis, the tracheal system and the posterior spiracles for Cht2, Cht7, Cht12 as well as idgf1, idgf3, and idgf6 (synonymous to Cht13 and DmDS47). The relative expression levels and the patterns correlated very well with phenotypes upon RNAi-mediated knockdown in the cuticle forming organs (Pesch et al., 2016).

However, systematic expression and subcellular localization analyses of individual Cht proteins and in particular for Cht2, which is crucial for larval development, are sparsely known. Here we show that the Cht2 protein is expressed in the cuticle forming organs resembling the Cht2 gene expression at the end of embryogenesis. Additionally, Cht2 staining was found in the secretory active salivary glands. Later on during larval development Cht 2 was detected in trachea, epidermis, spiracles and fore- and midgut, which all form various kinds of chitin-matrices, and in addition in imaginal discs and in parts of the salivary glands. Thus, the identified Cht2 protein expression pattern is consistent with the observation of Cht2 gene knockdown phenotypes leading to larval lethality, instable barrier of the body wall cuticle, molting defects of the larval mouth hooks and spiracles, pupal arrest and adult wing extension defects (Pesch et al., 2016). Our data further show that Cht2 is predominantly active in the cuticle forming organs but is additionally expressed in non-cuticular organs. This suggests that Cht2 may reach its target either by direct expression in cuticular organs or due to the secretion by the non-cuticular organs and the distribution via the hemolymph. The diversity of Cht2 expression obviously depends on the developmental steps and the requirement for processing of the chitin-matrix. The outer body wall cuticle is already established during late embryogenesis and needs further renewal and extension throughout larval development which correlates with constant epidermal Cht2 expression from late embryogenesis onwards. In contrast, the Cht 2 expression in the midgut may depend on functional properties of the organ, when food passage and digestion is needed during larval development.

Altogether these findings indicate diverse roles of Cht 2 at the various chitin-matrices. Cuticle molting defects upon RNAimediated knockdown suggest that one potential role could be the involvement of Cht2 in degradation of the old cuticle for larval molting (Pesch et al., 2016). Another, in the context of chitinolytic enzymes often discussed but rather speculative role, could be the Cht 2 requirement in the midgut for digestion of chitin-containing food. A third possible role of Cht 2 could be the involvement in the formation of chitinous structures. Here we present evidence that in Cht2 knockdown larvae the pore canal-like structures were not restricted to outer lamellae but appeared fragmented and misdistributed throughout the entire chitin-matrix. This could be due to deformed chitin-matrix architecture. Indeed, recent studies showed that the cuticle assembly zone, where chitin-matrix assembles and matures into compact lamellae, is strongly diminished and rudimentary in the Cht2 mutants (Pesch et al., 2016). This explains why the body wall procuticle failed to increase the number of chitin-lamellae and remained thin in the Cht2 knockdown (Fig. 4) (Pesch et al., 2016). Rapid production of a large number of chitinlamellae in $w t$ third instar larvae leads to the massive and thick procuticle at the end of larval development (Fig. 4A). In contrast, comparable Cht2 knockdown mutant larvae showed a thin body wall cuticle caused by inhibition of increasing the number of chitinmatrix-lamellae during intermolt (Fig. 4A). Thus, cuticle packaging, which is coordinated by the Obst-A core complex at the cuticle assembly zone and the chitin-lamellae requires Cht2. Our confocal localization data (Figs. 2 and 3 ) support these findings and show Cht2 enrichment at the apical surfaces of epithelial cells and in the epidermis additionally at the chitin-lamellae of the procuticle. Based on these findings we suggest various roles for Cht 2 in chitinmatrix formation (Fig. 5D). Cht2 may modify the variable length of nascent chitin-polymers, the elongation of fibers and the order of chitin-fibrils, which altogether supports the formation and preservation of the chitin-matrix by the Obst-A scaffold at the apical cell surface and at the procuticle. Any changes in the described processes may disturb the molecular mechanisms of chitin-matrix formation causing severe morphological defects, such as the absent cuticle assembly zone, the limited number of chitin-lamellae as well as the defective pore canal-like structure organization.

\section{Authors contributions}

Y.Y.P, D.R., M.B designed the experiments; Y.Y.P performed the experiments; D.R. did TEM studies, Y.Y.P, D.R., M.B. carried out data analysis. M.B. conceived and supervised the studies. M.B. developed the concept and wrote the manuscript.

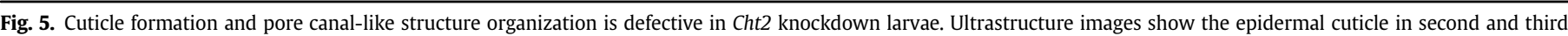

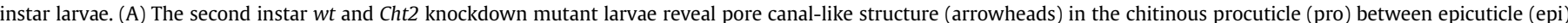

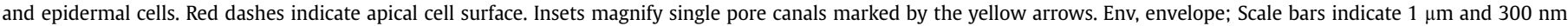

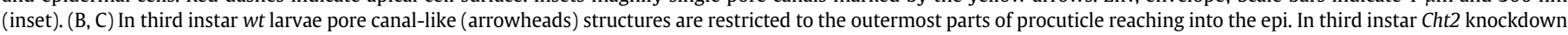

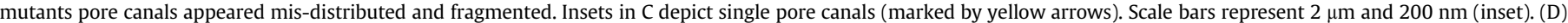


structure organization in third instar larvae. 


\section{Acknowledgements}

We thank the Bloomington and Vienna stock centers for providing fly stocks. We are grateful to Anne Uv for sharing flies. We are thankful to Yasmine Port and Lena Behr for critical reading. We appreciated technical support by Melanie Thielisch and Michelle Dassen. Special thanks go to Reinhard Bauer, Michael Hoch and Thomas Magin and the members of the departments for developmental biology in Bonn and Leipzig for scientific discussions and technical support. This work was supported by the DFG (SFB645 TPC5) to MB.

\section{References}

Adrangi, S., Faramarzi, M.A., 2013. From bacteria to human: a journey into the world of chitinases. Biotechnol. Adv. 31, 1786-1795.

Arakane, Y., Muthukrishnan, S., 2010. Insect chitinase and chitinase-like proteins. Cell. Mol. Life Sci. 67, 201-216.

Behr, M., Hoch, M., 2005. Identification of the novel evolutionary conserved obstructor multigene family in invertebrates. FEBS Lett. 579, 6827-6833.

Chandran, R., Williams, L., Hung, A., Nowlin, K., LaJeunesse, D., 2016. SEM characterization of anatomical variation in chitin organization in insect and arthropod cuticles. Micron 82, 74-85 (Oxford, England : 1993).

Chaudhari, S.S., Arakane, Y., Specht, C.A., Moussian, B., Boyle, D.L., Park, Y., Kramer, K.J., Beeman, R.W., Muthukrishnan, S., 2011. Knickkopf protein protects and organizes chitin in the newly synthesized insect exoskeleton. Proc. Natl. Acad. Sci. 108, 17028-17033.

Curtis, S., Sztepanacz, J.L., White, B.E., Dyer, K.A., Rundle, H.D., Mayer, P., 2013. Epicuticular compounds of Drosophila subquinaria and D. recens: identification, quantification, and their role in female mate choice. J. Chem. Ecol. 39, 579-590.

Dittmer, N.T., Tetreau, G., Cao, X., Jiang, H., Wang, P., Kanost, M.R., 2015. Annotation and expression analysis of cuticular proteins from the tobacco hornworm, Manduca sexta. Insect Biochem. Mol. Biol. 62, 100-113.

Galko, M.J., Krasnow, M.A., 2004. Cellular and genetic analysis of wound healing in Drosophila larvae. Plos Biol. 2, e239.

Gibbs, A.G., 1998. Water-proofing properties of cuticular lipids. Am. Zool. 38, $471-482$.

Jaspers, M.H.J., Pflanz, R., Riedel, D., Kawelke, S., Feussner, I., Schuh, R., 2014. The fatty acyl-CoA reductase waterproof mediates airway clearance in Drosophila. Dev. Biol. 385, 23-31.

Jasrapuria, S., Arakane, Y., Osman, G., Kramer, K.J., Beeman, R.W., Muthukrishnan, S., 2010. Genes encoding proteins with peritrophin A-type chitin-binding domains in Tribolium castaneum are grouped into three distinct families based on phylogeny, expression and function. Insect Biochem. Mol. Biol. 40, 214-227.

Jasrapuria, S., Specht, C.A., Kramer, K.J., Beeman, R.W., Muthukrishnan, S., Palli, S.R., 2012. Gene families of cuticular proteins analogous to peritrophins (CPAPs) in Tribolium castaneum have diverse functions. PLoS One 7, e49844.

Kawamura, K., Shibata, T., Saget, O., Peel, D., Bryant, P.J., 1999. A new family of growth factors produced by the fat body and active on Drosophila imaginal disc cells. Development 126, 211-219.

Kaznowski, C.E., Schneiderman, H.A., Bryant, P.J., 1985. Cuticle secretion during larval growth in Drosophila melanogaster. J. Insect Physiol. 31, 801-813.

Klocke, D., Schmitz, H., 2011. Water as a major modulator of the mechanical properties of insect cuticle. Acta Biomater. 7, 2935-2942.

Locke, M., 1961. Pore canals and related structures in insect cuticle. J. Biophys. Biochem. Cytol. 10, 589-618.

Luschnig, S., Bätz, T., Armbruster, K., Krasnow, M.A., 2006. Serpentine and vermiform encode matrix proteins with chitin binding and deacetylation domains that limit tracheal tube length in Drosophila. Curr. Biol. 16, 186-194.

Matsuda, Y., Osaki, T., Hashii, T., Koshiba, T., Kawabata, S., 2007. A cysteine-rich protein from an arthropod stabilizes clotting mesh and immobilizes bacteria at injury sites. J. Biol. Chem. 282, 33545-33552.

Merzendorfer, H., 2006. Insect chitin synthases: a review. J. Comp. Physiol. B 176, $1-15$.

Moussian, B., 2013. The apical plasma membrane of chitin-synthesizing epithelia. Insect Sci. 20, 139-146.
Moussian, B., Letizia, A., Martínez-Corrales, G., Rotstein, B., Casali, A., Llimargas, M. 2015. Deciphering the genetic programme triggering timely and spatiallyregulated chitin deposition. PLoS Genet. 11, e1004939.

Moussian, B., Tång, E., Tonning, A., Helms, S., Schwarz, H., Nüsslein-Volhard, C., Uv, A., 2006. Drosophila Knickkopf and retroactive are needed for epithelial tube growth and cuticle differentiation through their specific requirement for chitin filament organization. Development 133, 163-171.

Moussian, B., Uv, A.E., 2005. An ancient control of epithelial barrier formation and wound healing. Bioessays 27, 987-990.

Noh, M.Y., Kramer, K.J., Muthukrishnan, S., Kanost, M.R., Beeman, R.W., Arakane, Y. 2014. Two major cuticular proteins are required for assembly of horizontal laminae and vertical pore canals in rigid cuticle of Tribolium castaneum. Insect Biochem. Mol. Biol. 53, 22-29.

Pesch, Y.-Y., Riedel, D., Behr, M., 2015. Obstructor a organizes matrix assembly at the apical cell surface to promote enzymatic cuticle maturation in Drosophila. J. Biol. Chem. 290, 10071-10082.

Pesch, Y.-Y., Riedel, D., Patil, K.R., Loch, G., Behr, M., 2016. Chitinases and imaginal disc growth factors organize the extracellular matrix formation at barrier tissues in insects. Sci. Rep. 6, 18340.

Petkau, G., Wingen, C., Jussen, L.C.A., Radtke, T., Behr, M., 2012. Obstructor-A is required for epithelial extracellular matrix dynamics, exoskeleton function, and tubulogenesis. J. Biol. Chem. 287, 21396-21405.

Shaik, K.S., Pabst, M., Schwarz, H., Altmann, F., Moussian, B., 2011. The Alg5 ortholog Wollknauel is essential for correct epidermal differentiation during Drosophila late embryogenesis. Glycobiology 21, 743-756.

Stümpges, B., Behr, M., 2011. Time-specific regulation of airway clearance by the Drosophila J-domain transmembrane protein Wurst. FEBS Lett. 585, 3316-3321.

Tetreau, G., Dittmer, N.T., Cao, X., Agrawal, S., Chen, Y.R., Muthukrishnan, S., Haobo, J., Blissard, G.W., Kanost, M.R., Wang, P., 2015. Analysis of chitin-binding proteins from Manduca sexta provides new insights into evolution of peritrophin A-type chitin-binding domains in insects. Insect Biochem. Mol. Biol. 62, $127-141$.

Tonning, A., Hemphälä, J., Tång, E., Nannmark, U., Samakovlis, C., Uv, A., 2005. A transient luminal chitinous matrix is required to model epithelial tube diameter in the Drosophila trachea. Dev. Cell 9, 423-430.

Vincent, J.F.V., Wegst, U.G.K., 2004. Design and mechanical properties of insect cuticle. Arthropod Struct. Dev. 33, 187-199.

Wang, S., Jayaram, S.A., Hemphälä, J., Senti, K.-A., Tsarouhas, V., Jin, H., Samakovlis, C., 2006. Septate-junction-dependent luminal deposition of chitin deacetylases restricts tube elongation in the Drosophila trachea. Curr. Biol. 16, 180-185.

Willis, J.H., 2010. Structural cuticular proteins from arthropods: annotation, nomenclature, and sequence characteristics in the genomics era. Insect Biochem. Mol. Biol. 40, 189-204.

Wingen, C., Stümpges, B., Hoch, M., Behr, M., 2009. Expression and localization of clathrin heavy chain in Drosophila melanogaster. Gene Expr. Patterns 9, 549-554.

Wolfgang, W.J., Fristrom, D., Fristrom, J.W., 1987. An assembly zone antigen of the insect cuticle. Tissue Cell 19, 827-838.

Zhang, J., Zhang, X., Arakane, Y., Muthukrishnan, S., Kramer, K.J., Ma, E., Zhu, K.Y., 2011a. Comparative genomic analysis of chitinase and chitinase-like genes in the African malaria mosquito (Anopheles gambiae). PLoS One 6, e19899.

Zhang, J., Zhang, X., Arakane, Y., Muthukrishnan, S., Kramer, K.J., Ma, E., Zhu, K.Y., 2011 b. Identification and characterization of a novel chitinase-like gene cluster (AgCht5) possibly derived from tandem duplications in the African malaria mosquito. Anopheles gambiae. Insect Biochem. Mol. Biol. 41, 521-528.

Zhu, K.Y., Merzendorfer, H., Zhang, W., Zhang, J., Muthukrishnan, S., 2016. Biosynthesis, turnover, and functions of chitin in insects. Annu. Rev. Entomol. 61, 177-196.

Zhu, Q., Arakane, Y., Banerjee, D., Beeman, R.W., Kramer, K.J., Muthukrishnan, S., 2008a. Domain organization and phylogenetic analysis of the chitinase-like family of proteins in three species of insects. Insect Biochem. Mol. Biol. 38, 452-466.

Zhu, Q., Arakane, Y., Beeman, R.W., Kramer, K.J., Muthukrishnan, S., 2008b. Characterization of recombinant chitinase-like proteins of Drosophila melanogaster and Tribolium castaneum. Insect Biochem. Mol. Biol. 38, 467-477.

Zhu, Q., Arakane, Y., Beeman, R.W., Kramer, K.J., Muthukrishnan, S., 2008c. Functional specialization among insect chitinase family genes revealed by RNA interference. Proc. Natl. Acad. Sci. 105, 6650-6655. 